\title{
Latent Curve Analyses of Longitudinal Twin Data Using a Mixed-Effects Biometric Approach
}

\author{
John J. McArdle \\ Department of Psychology, University of Southern California, Los Angeles, United States of America
}

In a recent article McArdle and Prescott (2005) showed how simultaneous estimation of the biometric parameters can be easily programmed using current mixed-effects modeling programs (e.g., SAS PROC MIXED). This article extends these concepts to deal with mixed-effect modeling of longitudinal twin data. The biometric basis of a polynomial growth curve model was used by Vandenberg and Falkner (1965) and this general class of longitudinal models was represented in structural equation form as a latent curve model by McArdle (1986). The new mixed-effects modeling approach presented here makes it easy to analyze longitudinal growth-decline models with biometric components based on standard maximum likelihood estimation and standard indices of goodness-of-fit (i.e., $\chi^{2}, d f, \varepsilon_{a}$ ). The validity of this approach is first checked by the creation of simulated longitudinal twin data followed by numerical analysis using different computer programs (i.e., Mplus, Mx, MIXED, NLMIXED). The practical utility of this approach is examined through the application of these techniques to real longitudinal data from the Swedish Adoption/Twin Study of Aging (Pedersen et al., 2002). This approach generally allows researchers to explore the genetic and nongenetic basis of the latent status and latent changes in longitudinal scores in the absence of measurement error. These results show the mixed-effects approach easily accounts for complex patterns of incomplete longitudinal or twin pair data. The results also show this approach easily allows a variety of complex latent basis curves, such as the use of age-at-testing instead of wave-oftesting. Natural extensions of this mixed-effects longitudinal approach include more intensive studies of the available data, the analysis of categorical longitudinal data, and mixtures of latent growth-survival/ frailty models.

Much of the recent statistical literature in behavioral science uses mixed-effects multilevel algorithms (MEMA; e.g., Bryk \& Raudenbush, 1992; Goldstein, 1995; McArdle \& Hamagami, 1996). The MEMA calculation can be seen as a variance components approach with the added potential of a restricted structure on the means (i.e., as in repeated measures ANOVA). In a recent paper Guo and Wang (2002) described some interesting behavior genetic type applications of MEMA, including modeling with multivariable family data (using SAS PROC MIXED). However, simultaneous estimates of the key parameters representing genetic and nongenetic sources of variation among individuals from twin data were not included in their approach. Subsequently, McArdle and Prescott (2005) showed how simultaneous estimation of the biometric parameters can be easily programmed using current MEMA programs (e.g., SAS PROC MIXED). The communalities among path analysis models (PAM) and variance component models (VCM) fitted using structural equation modeling algorithms (SEMA) suggested ways standard MEMA programs could be constructed to yield identical statistical information and substantive inferences. This offered an easy way to include measured covariates, observed variable interactions, and multiple relatives within each family. Available software such as Mplus (Muthén \& Muthén, 2002; Prescott, 2004), Mx (Neale et al., 1999), and PROC MIXED (Littell et al., 1996; SAS, 1991) were compared for programming complex models, including the flexibility of data input, treatment of missing data, inclusion of covariates, and ease of accommodating varying numbers of observations (per family or individual). This convergence of techniques showed alternative ways researchers using behavior genetic designs can choose alternative models and programs, and suggested different options would be useful for different kind of problems.

This article extends these biometric concepts and deals with mixed-effect multilevel modeling concepts for longitudinal twin data. The biometric basis of a

Received 19 December, 2005; accepted 16 January, 2006.

Address for correspondence: John J. McArdle, National Growth and Change Study Laboratory, Department of Psychology, SGM 824, University of Southern California, Los Angeles, CA 90089, USA. E-mail:jmcardle@usc.edu 


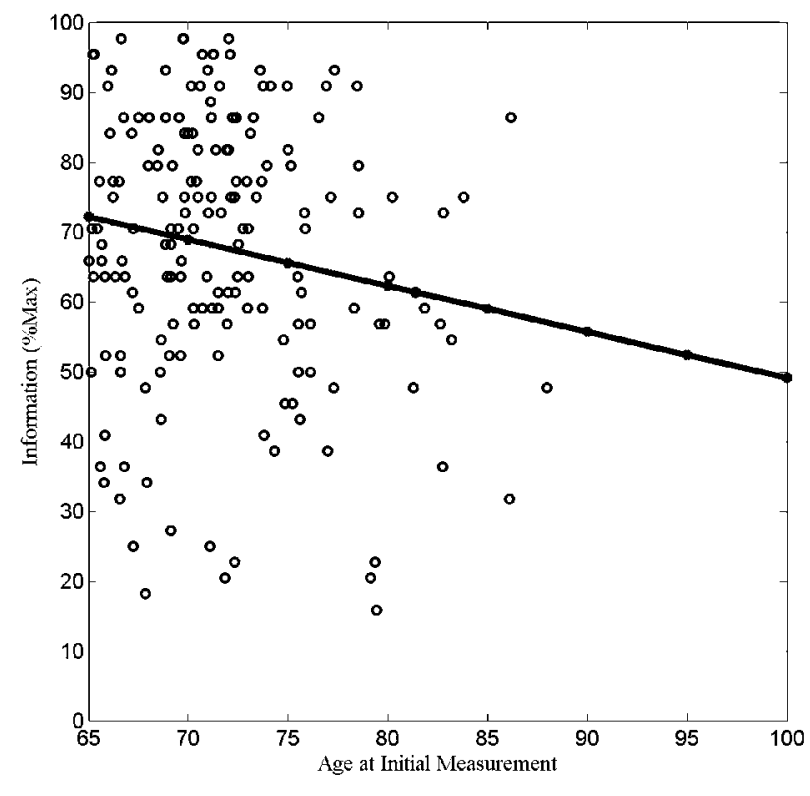

Figure 1a

A plot of cross-sectional data of the Information Subscale at Wave 1 in SATSA (age $\geq 65$, one person per family).

polynomial growth curve model was used by Vandenberg and Falkner (1965) and this general class of longitudinal models was represented as a latent curve model (LCM) by McArdle (1986). The benefits of writing the LCM as structural equation modeling (SEM) include the use of available SEMA software (e.g., LISREL by Jöreskog \& Sörbom, 1984), and computing of standard maximum likelihood estimation (MLE) and standard indices of goodness-of-fit (i.e., $\chi^{2}, d f, \varepsilon_{\mathrm{a}}$ ). Over the past 20 years there have been several substantive applications of this LCM-twin approach (e.g., Finkel at al., 2003; Johansson et al., 2004; McArdle et al., 1998; McGue \& Christensen, 2002; Reynolds et al., 2005), and further methodological research (e.g., McArdle \& Hamagami, 2003; Neale \& McArdle, 2000). Other longitudinal SEM based primarily on autoregressive structures have been considered as well (Baker et al., 1983; Boomsma et al., 1989; Dolan et al., 1991).

In the first section of this article we overview the basic MEMA equations for twin data, show how the standard longitudinal LCM can be reparameterized in terms of orthogonal variance components, and then combine both approaches into a LCM-twin model. This clarifies how we can interpret and make inferences about the biometric components of change in longitudinal twin analysis. Simulated longitudinal twin data are used to illustrate the required programming (scripts available) and examine the numerical outcomes of different computer programs (i.e., Mplus, MIXED, NLMIXED).

In the second section we demonstrate the utility of these models by applying this approach to longitudinal data from the Swedish Adoption/Twin Study of

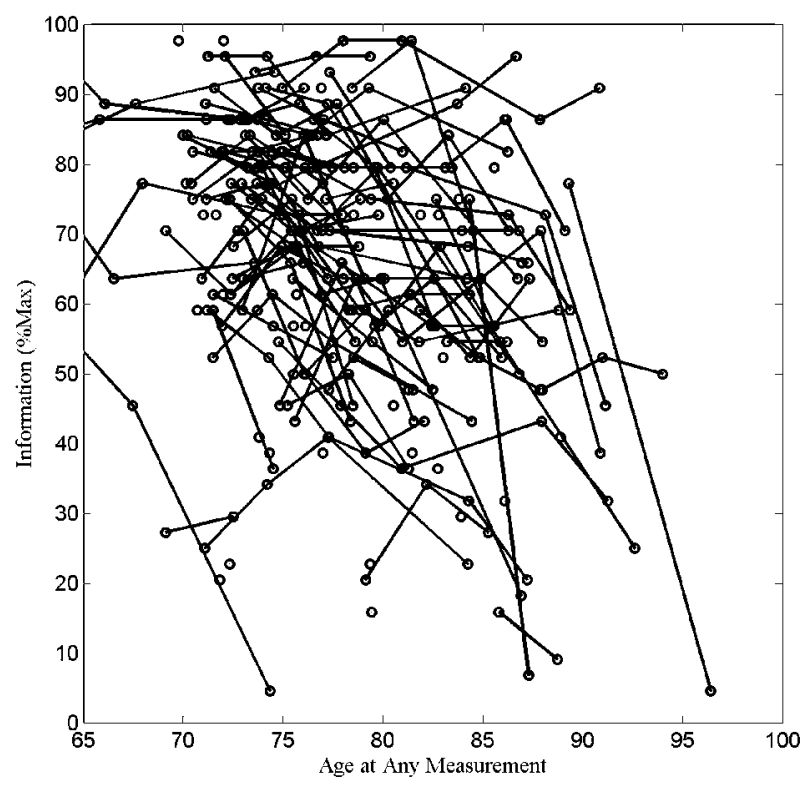

Figure 1b

A plot of longitudinal trajectories of the Information Subscale over five waves in SATSA ( $50 \%$ random sample, one person per family).

Aging (SATSA; Pedersen et al., 2002). The kinds of problems considered here are illustrated in the plots of Figure 1 using SATSA data. To simplify these plots we only present a $50 \%$ random sample of families and only one person from each family. The first Figure $1 \mathrm{a}$ is a cross-sectional scatter plot of the scores on Age and Information scores at the first wave for a sample of persons (mean age 71.8, range 65-88). A simple linear regression line is included to show a small age decline found in the Information score at these ages (but $R^{2} \sim$ $.02)$. In contrast, Figure $1 \mathrm{~b}$ is a longitudinal trajectory plot of the Information score at the age of measurement across up to five waves of testing. These data are collected over five waves of testing but these individual trajectories are plotted over age-of-testing to match the cross-sectional age regression (see McArdle \& Bell, 2000; McArdle et al., 2005). This shows a wide range of scores at most ages, a tendency for the decline over age, and many different individual trajectories with a limited number of scores for any individual.

This real and complex data collection poses many challenges for longitudinal and behavior genetic research. The purpose of this article is to show how many common longitudinal twin problems can be easily handled using a biometric mixed-effects approach. One problem is dealing with incomplete longitudinal or twin pair data, and here attrition over time and within pairs is dealt with using missing-at-random (MAR) techniques for incomplete and sampling bias correction (e.g., McArdle et al., 1998; cf., Pedersen et al., 2003). This mixed-effects approach is flexible and permits the examination of a wide variety of incomplete latent basis curves, including the use of the age-at-testing as the 
basis for each individual (e.g., Finkel et al., 2003; McArdle et al., 1998). Since SATSA includes twins reared apart, we considering additional biometric components for rearing environments. A final practical problem is the calculation and interpretation of level and slope parameters over age, and one such approach (after McArdle et al., 1998) is automated by standard computer scripts (SAS).

\section{Mixed Effects and Longitudinal Twin Models Mixed-Effect Biometric Variance Component Models}

In traditional biometric theory we write the observed score $\left(Y_{n}\right)$ for any person $(n=1$ to $N)$. Here we include different families $(f=1$ to $F)$ and different individuals $\left(I=1\right.$ to $\left.I_{f}\right)$ within the family. We then write a structural equation model for any pair of persons as

$$
\begin{aligned}
Y_{f, 1} & =\mu+A_{f, 1}+S_{f}+E_{f, 1} \\
& =\mu+\sigma_{a} a_{f 1}+\sigma_{s} s_{f}+\sigma_{e} e_{f 1}, \text { and } \\
Y_{f, 2} & =\mu+A_{f, 2}+S_{f}+E_{f, 2} \\
& =\mu+\sigma_{a} a_{f 2}+\sigma_{s} s_{f}+\sigma_{e} e_{f 2}, \text { with } \\
E\left\{a_{1}, a_{2}\right\}=\rho_{a} . &
\end{aligned}
$$

In this classic expression we include the mean $(\mu)$ and three independent sources of the deviations around the mean. These are deviations due to additive genetic differences $(A)$, deviations that are nongenetic but shared by family members $(S)$, and deviations due to nongenetic factors specific to the individual $(E)$. (Note: We use the term $S$ here because we reserve $C$ to define 'common' components in many subsequent models.) These deviations can also be written with unobserved scores scaled to unit variance $(E\{a, a\}=E\{s, s\}=$ $E\{e, e\}=1)$ so the coefficients $\left(\sigma_{i}\right)$ represent the standard deviation of each component, and the genetic correlation is assigned based on the genetic relationship of the pair (e.g., for monozygotic $[M Z]$ pairs, $\rho_{\mathrm{a}}=1$, whereas for dizygotic [DZ] pairs, $\rho_{a}=1 / 2$ ). In the simplest version of biometric theory we assume these components are all uncorrelated within a person. More complex versions of this model include components to represent special environments, nonadditivity (e.g., dominance deviations), correlations among components (e.g., $\rho\{A, E\} \neq 0$ ), and interactions among components (e.g., $A$ by $E$ ).

The standard model for a pair of relatives is drawn as a latent variable path diagram using the notation of Figure 2a. Most features of this path diagram are familiar, but it is useful to point out that in this particular diagram: (a) all paths that are not explicitly labeled are equal to unity; (b) the variance terms are represented as standard deviations on paths, (c) the means are one-headed arrows from the unit constant and are set equal over all persons, (d) the variances are two-headed arrows on a variable and are fixed at 1 , and (e) the covariances are two-headed arrows connecting two variables and may differ for different groups of relatives.

In the mixed-effects approach used by McArdle and Prescott (2005) all paths are known values for each person. We can rewrite Equations 1 for a pair of persons within the same family as

$$
\begin{aligned}
& Y_{f, 1}=\mu+A_{f 1}+S_{f}+E_{f 1} \text {, with } A_{f 1}=w_{a c} A C_{f}+w_{a u} A U_{f 1}, \\
& \text { and } \\
& Y_{f, 2}=\mu+A_{f 2}+S_{f}+E_{f 2} \text {, with } A_{f 2}=w_{a c} A C_{f}+w_{a u} A U_{f 2},
\end{aligned}
$$

where the additive genetic deviation is separated into two deviation scores: $A C_{f}$ is common for members of the same family, and $A U_{f i}$ is unique to the individual. In this expression the weights $(W)$ are fixed at values which indicate the proportion of the additive genetic deviation shared between relatives. This general variance components model is drawn following Equations 2 as a nested or higher-order latent variable path diagram in Figure $2 \mathrm{~b}$. It can also be written in reduced form by substituting the equation for $A_{f}$ back in the equation for $Y_{f}$.

The reparameterization of Equations 1 into the equivalent model in Equations 2 is based on the representation of two correlated factors as a single shared factor with two unique factors. The move from standard path model (1a) to mixed-effect path model (1b) is based on the classical separation of the additive genetic variability for each individual $\left(A_{1}\right.$ and $\left.A_{2}\right)$ into two parts - one part that is common to both members of the pair $(A C)$ and the one part that is unique to each individual $\left(A U_{1}\right.$ and $\left.A U_{2}\right)$. In theory, this separation of additive genetic deviations into common and unique components reflects the result of transmission of parental alleles to offspring. Under standard assumptions of assortative mating, each of these two new components is assigned the same genetic variance $\left(\sigma_{a}^{2}\right)$, we assume these two scores are uncorrelated $(\rho\{A C, A U\}=0)$, and the weights $(W)$ are separated into those which are common to the family $\left(w_{a c}\right)$ and specific to the individual $\left(w_{\text {au }}\right)$. In this formulation, the typical additive genetic correlation $\left(r_{a}\right)$ is not included and there are no resulting covariance terms.

The computational approach suggested by McArdle and Prescott (2005; termed Script 1) was based on this reparameterization of the usual path model (Figure 2a) so the paths (coefficients) are all fixed weights defined by the application (Figure 2b). To ensure these weights add no extra impacts on the estimation of the variance terms, they are scaled so their sum of squares is unity $\left(w_{a c}^{2}+w_{a u}^{2}=1\right)$. Since the two individuals in any MZ pair are assumed to share the same genotypes, we simplify the model for an MZ pair by fixing $w_{a c}=1$ and $w_{a u}=0$ (in Equations 2). In contrast, the assumption of no assortative mating implies that members of DZ pairs share half of their genotypes (due to segregation of alleles), and this implication can be represented as fixed weights of $w_{a c}=\sqrt{1 / 2}$ and $w_{a u}=\sqrt{1 / 2}$ (in Equations 2). In order to estimate genetic dominance we add two new latent scores, $D_{f, i}$ for each individual, decomposed into common $D C$ and unique $D U_{f i}$, with both assigned 


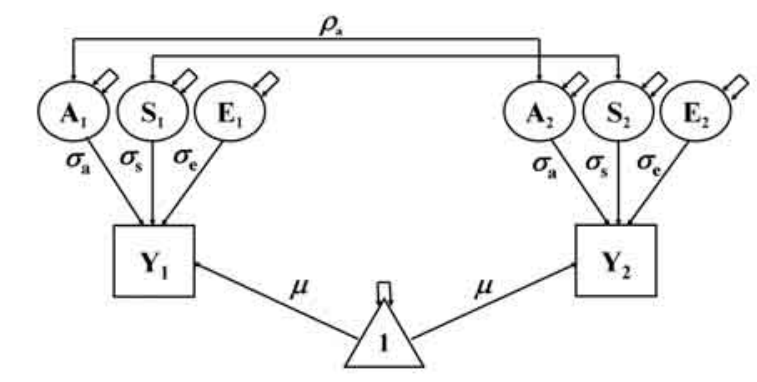

Figure 2a

A standard path diagram of a twin model including means.

Note: Parameters not labeled are fixed at $1 ; r a=1$ for $M Z$ pairs and $r a=.5$ for $D Z$ pairs.

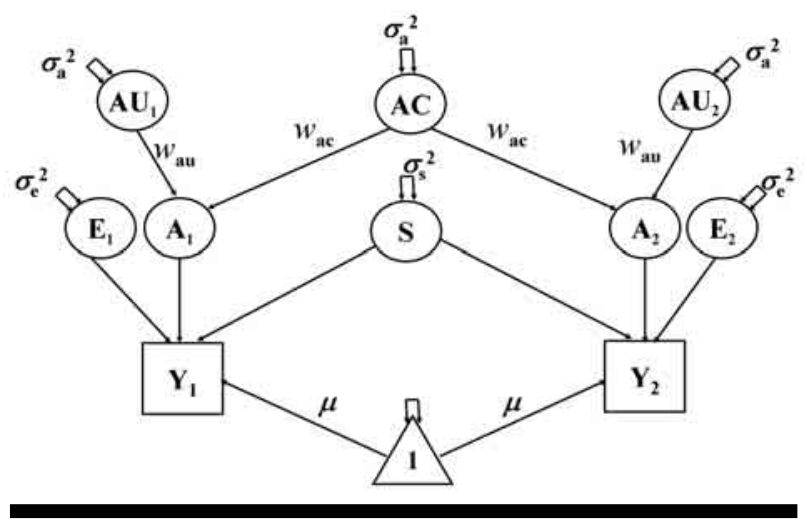

Figure 2b

SEM representation of the computational basis of a mixed-effects multilevel model based on orthogonal variance components (from McArdle \& Prescott, 2005).

equal variance (i.e., $\left.\sigma_{d}^{2}\right)$. The analysis of MZ-DZ twins would require corresponding weights of 1 and 0 in $M Z$ pairs and weights of $\sqrt{1 / 4}$ and $\sqrt{3 / 4}$ and for $D C$ and unique $D U_{f i}$ respectively. Similar terms can be added to deal with special features of the data collection design (e.g., rearing, adoption; see Cattell, 1982; McArdle \& Prescott, 2005).

\section{Latent Curves as Mixed-Effects Models}

We now extend the path analysis and variance components models to a longitudinal curve model for twin data. In these analyses we are interested in the trajectory over time as well as the nested structure of the correlations among relatives, so we present the model needed to estimate all the parameters of the model both longitudinal and biometric. One generic form of a LCM based on a trajectory over time for observed variables is written as

$$
Y[\mathrm{t}]_{\mathrm{n}}=G 0_{n}+\alpha[\mathrm{t}] G 1_{\mathrm{n}}+U[\mathrm{t}]_{\mathrm{n}},
$$

where for any individual $(n=1$ to $N)$ we write three unobserved latent variables: (1) G0 are termed the initial level scores, (2) G1 are termed the slope scores, and (3) $U[t]$ are unobserved and independent unique errors for measurements at each occasion. The basis coefficients $\alpha[t]$ are weights used to represents some function of the timing of the observations. In some cases the elements of this vector are assigned $\alpha[1]=0$, $\alpha[2]=1, \alpha[3]=2$, and $\alpha[4]=3$, to represent a linear trend over the waves of measurement. In more complex cases we can fix $\alpha[\mathrm{t}]=f\{$ Age $[\mathrm{t}]\}$ to represent the age at the time of the observation (as in McArdle et al., 1998).

The typical application of this latent growth model further presumes that

$$
\begin{aligned}
& G 0_{n}=\beta_{o}+D 0_{n}, \\
& G 1_{n}=\beta_{o}+D 1_{n}^{n} \text {, and } \\
& E\{D 0, D 1\}=\sigma_{01}
\end{aligned}
$$

so the initial level and slopes are assumed to be random variables with 'fixed' means $\left(\beta_{0}, \beta_{1}\right)$ but with unobserved deviations $(D 0, D 1)$ that have 'random' variances $\left(\sigma_{0}^{2}, \sigma_{1}^{2}\right)$ and covariance $\left(\sigma_{01}\right)$. The path diagram of Figure $3 \mathrm{a}$ is a standard representation of this kind of latent growth model. Model parameters representing 'fixed' or 'group' coefficients are drawn as one-headed arrows while 'random' or 'individual' features are drawn as two-headed arrows. The unique error terms are assumed to be normally distributed with mean zero and constant variance $\left(\sigma_{u}^{2}\right)$ and uncorrelated with all other components. It is now well known that these models can be fitted using either standard SEM path analysis approach (McArdle \& Bell, 2000) or using standard mixed-effects approach (e.g., see Script 3a; Verbeke \& Molenberghs, 2000; Pinheiro \& Bates, 2000). Some differences between two approaches have been found in the practical ease of representing different basis functions (i.e., exact ages, nonlinear curves, free basis parameters).

The covariance of the initial level and slope is an important parameter in the typical LCM because it permits the placement of the initial level at any position without restricting model fit and substantive interpretation. This covariance can equivalently be represented as a regression of slope on level $\left.\beta_{10}\right)$ or level on slope $\left(\beta_{01}\right)$. However, it is possible to rewrite these standard LCM assumptions in the somewhat nonstandard form of

$$
\begin{aligned}
& G O_{n}=\beta_{o}+C 01_{n}+U 0_{n} \\
& G 1_{n}=\beta_{1}+C 01_{n}+U 1_{n}, \text { and } \\
& E\{U O, U 1\}=0 .
\end{aligned}
$$

In this expression we add a new latent score C01, which is interpreted as a common factor of level and slope, with uncorrelated unique scores $(U 0, U 1)$. Now by simple substitution, we can rewrite the reduced form of the basic growth model (3) as

$$
\begin{aligned}
Y[\mathrm{t}]_{n} & =\left(C 01_{n}+U 0_{n}\right)+\alpha[\mathrm{t}]\left(C 01_{n}+U 1_{n}\right)+U[\mathrm{t}]_{n} \\
& =U 0_{n}+\alpha[\mathrm{t}] U 1_{n}+C 01_{n}+\alpha[\mathrm{t}] C 01_{n}+U[\mathrm{t}]_{n} \quad[6] \\
& =U 0_{n}+\alpha[\mathrm{t}] U 1_{n}+(1+\alpha[\mathrm{t}]) C 01_{n}+U[\mathrm{t}]_{n} .
\end{aligned}
$$

This expresses this LCM as a sum of four independent components. There are now two basis coefficients $(\alpha[t]$ and $1+\alpha[t])$ but all the model components (C01 UO, $U 1, U[t])$ are presumed to be uncorrelated. Most critically, this reduced form of the LCM equation 


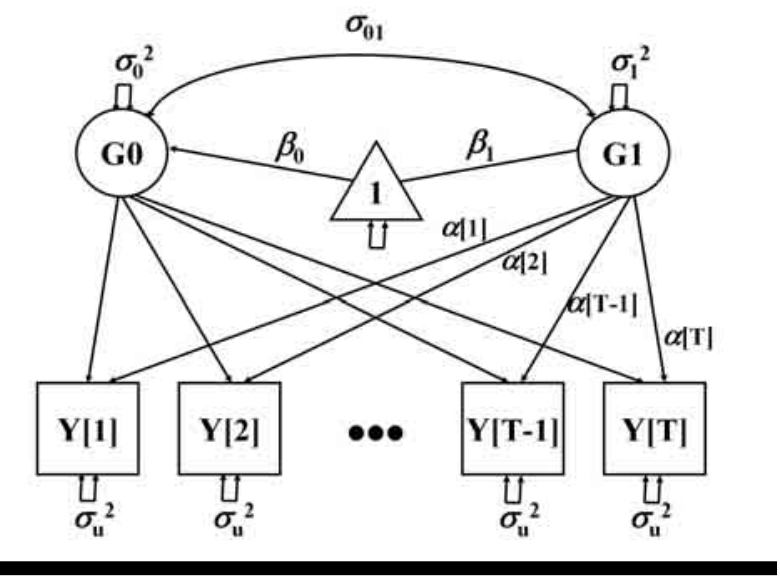

Figure 3a

A latent growth model represented in path analysis form (after McArdle, 1986).

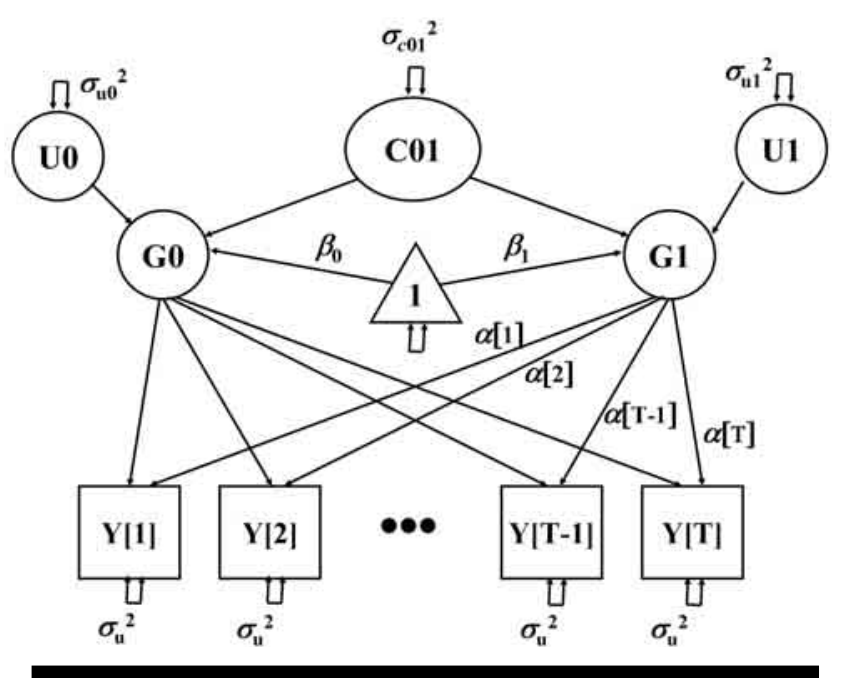

Figure $\mathbf{3 b}$

A path diagram of the latent curve model for longitudinal data represented as orthogonal variance components (all nonlabeled paths fixed at 1).

creates a set of orthogonal variance components and this is drawn in Figure $3 b$.

As it turns out, the LGM written as Equation 6 is especially practical for programming correlated latent variables as orthogonal variance components (e.g., see Script $3 \mathrm{~b}$ ). As in earlier models, the variance of any component is the sum of the variance that is common and the variance that is unique. For example, we can now reconstruct any of the standard unstructured variance and covariance parameters simply by writing

$$
\begin{aligned}
& \sigma_{0}^{2}=\sigma_{c 01}{ }^{2}+\sigma_{u 0}^{2}, \\
& \sigma_{1}^{2}=\sigma_{c 01}{ }^{2}+\sigma_{u 1}^{2}, \\
& \sigma_{01}=\sigma_{c 01}{ }^{2}, \text { and } \\
& \sigma_{Y[t]}{ }^{2}=\sigma_{u 0}{ }^{2}+\alpha[t]^{2} \sigma_{u 1}{ }^{2}+(1+\alpha[t])^{2} \sigma_{c 01}{ }^{2}+\sigma_{u[t]}^{2} .
\end{aligned}
$$

This orthogonal decomposition is one way to express the complex contribution of the correlation of the level and the slope to the variation at any time point.
This also means that negative variance components (e.g., $\sigma_{c 01}{ }^{2}<0$ ) should be informative in some cases.

The use of an orthogonal-reduced form of the basic growth model (Equations 3 to 7) is a general approach that can be used with any form of latent growth curve. For example, any LCM with a second basis function can be used for quadratic polynomials or the inclusion of practice effects (e.g., McArdle \& Woodcock, 1997). To add a second basis coefficients we write

$$
\begin{aligned}
Y & {[\mathrm{t}]_{\mathrm{n}}=G 0_{n}+\alpha[\mathrm{t}] G 1_{\mathrm{n}}+\gamma[\mathrm{t}] G 2_{\mathrm{n}}+U[\mathrm{t}]_{\mathrm{n}}, } \\
= & \left(\mathrm{C} 01_{n}+C 02_{n}+U 0_{n}\right)+\alpha[\mathrm{t}]\left(C 01_{n}+C 12_{n}+U 1_{n}\right) \\
& +\gamma[\mathrm{t}]\left(C 02_{n}+C 12_{n}+U 2_{n}\right)+U[\mathrm{t}]_{n} \\
= & U 0_{n}+\alpha[\mathrm{t}] U 1_{n}+\gamma[\mathrm{t}] U 2_{n}+C 01_{n}+\alpha[\mathrm{t}] C 01_{n} \\
& +C 02_{n}+\gamma[\mathrm{t}] C 02_{n}+\alpha[\mathrm{t}] C 12_{n}+\gamma[\mathrm{t}] C 12_{n}+U[\mathrm{t}]_{n} \\
= & U 0_{n}+\alpha[\mathrm{t}] U 1_{n}+\gamma[\mathrm{t}] U 2_{n}+(1+\alpha[\mathrm{t}]) C 01_{n} \\
& +(1+\gamma[\mathrm{t}]) C 02_{n}+(\alpha[\mathrm{t}]+\gamma[\mathrm{t}]) C 12_{n}+U[\mathrm{t}]_{n} .
\end{aligned}
$$

This reduced form expresses a dual basis LCM with two correlated components $(G 0, G 1)$ and one unique term $(u[\mathrm{t}])$ as a sum of seven orthogonal components (U0, U1, U2, C01, C02, C12, and $U[t])$. Of course, instead of two sets of basis coefficients $(\alpha[t]$ and $\gamma[t])$ this model requires five sets of related basis coefficients $(\alpha[t], 1+\alpha[t], \gamma[t], 1+\gamma[t]$, and $\alpha[t]+\gamma[t])$. If the basis coefficients are set at known values the setup of these five basis functions is a trivial programming problem. If we further restrict the second basis to be a function of the first, we can obtain models such as an orthogonal form of the classic quadratic polynomial model (e.g., $\gamma[t]=1 / 2 \alpha[t]^{2}$; Finkel et al., 2003; Reynolds et al., 2005; Vandenberg \& Falkner, 1965). In all cases, the reconstruction of the variance and covariances from the orthogonal variance components is carried out in the same simple additive fashion (e.g., Equations $7 \mathrm{~b}$ ).

\section{Longitudinal Biometric Variance Component Models}

We next represent the biometric decomposition of the latent components of level and slope following McArdle (1986). First, we assume the longitudinal growth representation of Equation 5 is appropriate for all individuals. Next, using pairs of relatives, we consider the initial level to be decomposable as

$$
\begin{aligned}
& G O_{f, 1}=\beta_{0}+A O_{1}+S O_{f}+E O_{1}, \\
& G O_{f, 2}=\beta_{0}+A O_{2}+S O_{f}+E O_{2} \text {, and } \\
& E\left\{A O_{1}, A O_{2}\right\}=\rho_{a} \sigma_{0 a}{ }^{2}
\end{aligned}
$$

where three new latent variables are added within each person $\left(\mathrm{AO}_{n}, \mathrm{SO}_{f}, E \mathrm{O}_{n}\right)$ and where the genetic correlation is assigned for the group (e.g., $\rho_{a}=1$ or $\rho_{a}=1 / 2$ ). In this way, the biometric parameters $\left(\sigma_{0 a}^{2}, \sigma_{0 s}^{2}, \sigma_{0 e}^{2}\right)$ indicate features of the latent initial level variance. We write the same model for the latent slopes across pairs as

$$
\begin{aligned}
& G 1_{f, 1}=\beta_{1}+A 1_{1}+S 1_{f}+E 1_{1}, \\
& G 1_{f, 2}=\beta_{1}+A 1_{2}+S 1_{f}+E 1_{2}, \text { and } \\
& E\left\{A 1_{1}, A 1_{2}\right\}=r_{a} \sigma_{1 a}^{2} .
\end{aligned}
$$


To decompose the covariance we can rewrite the score model (5) to include a regression on the initial level latent variables, although we recognize this choice of the 'slope as an outcome' is usually arbitrary (as in McArdle et al., 1998). A path analysis diagram of this model is presented in Figure $4 \mathrm{a}$ and, as shown in many other research reports, this model can be fitted as a structural equation model with multiple groups. One intentional limitation of this approach is that the means over time represent age changes at the phenotypic level while the deviations around these means represent the influences of genetic and nongenetic sources (cf., Dolan et al., 1991).

To fit this same model using MEMA techniques we can adapt the orthogonal variance components approach established in Equation 6. We now reparameterize both common and unique parts of these components as

$$
\begin{aligned}
& Y[\mathrm{t}]_{f, i}=U 0_{i}+\alpha[\mathrm{t}] U 1+(1+\alpha[\mathrm{t}]) C 01_{i}+U[\mathrm{t}]_{i}, \\
& U 0_{f, i}=\beta_{0}+A 0_{i}+S 0_{f}+E 0_{i}, \\
& U 1_{f, i}=\beta_{1}+A 1_{i}+S 1_{f}+E 1_{i}, \text { and } \\
& C 01_{f, I}=A 01_{i}+S 01_{f}+E 01_{i} .
\end{aligned}
$$

To be consistent with the previous latent curve twin path model, we do not permit a mean for the common factor C01, and we do not attempt to decompose the residual error variance $\left(\sigma_{u}^{2}\right.$, within a person at a specific occasion). To specify the model as a set of orthogonal variance components, we rewrite Equation 11 in terms of fixed weights and in the reduced form of

$$
\begin{aligned}
& Y[\mathrm{t}]_{f, i}=U 0_{i}+\alpha[\mathrm{t}] U 1+(1+\alpha[\mathrm{t}]) C 01_{i}+U[\mathrm{t}]_{i}, \\
& U 0_{f, i}=\beta_{0}+A u 0_{f, i}+\operatorname{Su} 0_{f}+E u 0_{i}, \\
& A u 0_{f, i}=w_{a c} A C u 0_{f}+w_{a u} A U u 0_{i}, \\
& U 1_{f, i}=\beta_{1}+A u 1_{f, i}+S u 1_{f}+E u 1_{i,}, \\
& A u 1_{f, i}=w_{a c} A C u 1_{f}+w_{a u} A U u 1_{i}, \text { and } \\
& C 01_{f, i}=A 01_{f, i}+S 01_{f}+E 01_{i}, \\
& A 01_{f, i}=w_{a c} A C 01_{f}+w_{a u} A U 01_{i} .
\end{aligned}
$$

In this model the additive genetic weights $\left(w_{a c}\right.$ and $\left.w_{a u}\right)$ are defined based on the genetic resemblance of the pairs (e.g., $W_{m z}=[1,0], W_{d z}=[\sqrt{1 / 2}, \sqrt{1 / 2}]$ ), we estimate two means, one error variance, and nine orthogonal variance components (three which represent covariance information). The higher-order version of the orthogonal model is portrayed in Figure $4 \mathrm{~b}$. Of course, this is an unusual looking path diagram, but it attempts to represent Equation 12 exactly. This includes having the means for the unique components (but not the C01) although if we put the means on the level and slope the model expectations would be the same. Most importantly, this SEM offers a direct way to fit a longitudinal twin model using standard mixedeffects MEMA techniques (see Script 4).

Proportions of variance are often calculated in biometric genetic analyses, but this can be a complex problem when dealing with different variables or time points. One issue that needs to be considered is the

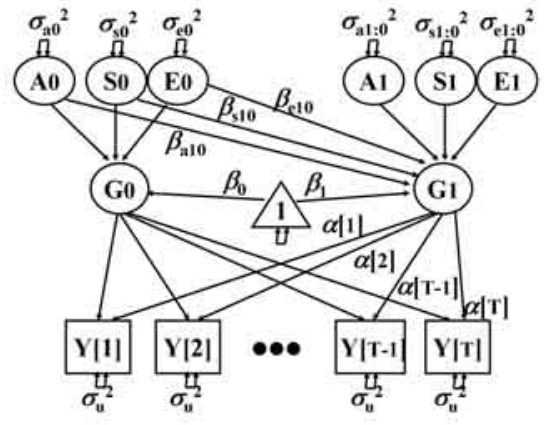

Figure 4a

A one-group path diagram of an latent curve model for longitudinal twin data (e.g., McArdle, 1986; McArdle et al., 1998).

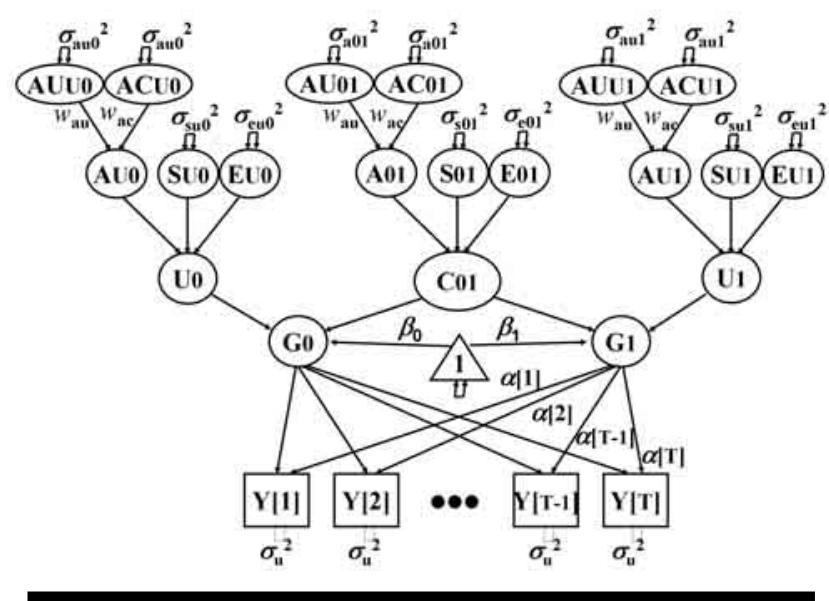

\section{Figure $4 b$}

A path diagram of the latent curve model for longitudinal pairs represented as orthogonal variance components (one person only, all nonlabeled paths fixed at 1, all weights $w$ determined by pair relationships).

changing baseline of expected variance at each age. Another issue is to consider how the addition of the independent and common components of the slope combine to form the change variance (see McArdle \& Woodcock, 1997). One way to deal with this longitudinal problem is to compare the changes in the expected variances at specific ages (as done in McArdle, 1986; McArdle \& Hamagami, 2003; McArdle et al., 1998). Using the basic terms presented in the earlier models we can now write the variance at any time point in terms of orthogonal components

$$
\begin{aligned}
& \sigma_{u 0}{ }^{2}=\sigma_{a u 0}{ }^{2}+\sigma_{s u 0}{ }^{2}+\sigma_{e u 0}{ }^{2}, \\
& \sigma_{u 1}{ }^{2}=\sigma_{a u 1}{ }^{2}+\sigma_{s u 1}{ }^{2}+\sigma_{e u 1}{ }^{2}, \\
& \sigma_{c 01}{ }^{2}=\sigma_{a 01}{ }^{2}+\sigma_{s 01}{ }^{2}+\sigma_{e 01}{ }^{2} \text {, and } \\
& \sigma_{Y[t]}{ }^{2}=\sigma_{u 0}{ }^{2}+\alpha[\mathrm{t}]^{2}{\sigma_{u 1}}^{2}+(1+\alpha[\mathrm{t}])^{2}{\sigma_{c o 1}}^{2}+\sigma_{u[\mathrm{t}]}{ }^{2} .
\end{aligned}
$$

Once again, this orthogonal decomposition is a simple way to express the otherwise complex contribution of the correlation of the level and the slope to the variation at any time point, and this is easy to program (see Script 4). It is now possible to investigate the relative 
contributions of the biometric parameters to any specific aspect of the total variation.

\section{Programming Longitudinal Biometric Mixed-Effect Models}

To compare the applications of the approaches described above we generated a set of longitudinal twin data based on a population model with the following structure:

1. Phenotypic Components: $T=5, \beta_{0}=25, \beta_{1}=10$, $\sigma_{u}^{2}=50$;

2. Biometric Structure of Initial Level: $\sigma_{a u 0}^{2}=50$, $\sigma_{s u 0}{ }^{2}=0, \sigma_{e u 0}{ }^{2}=50$;

3. Biometric Structure of Linear Slope: $\sigma_{a u 1}{ }^{2}=0$, $\sigma_{s u 1}^{2}=5, \sigma_{e u 1}^{2}=5$;

4. Biometric Structure of Level-Slope Covariation: $\sigma_{a 01}^{2}=0, \sigma_{s 01}^{2}=0, \sigma_{e 01}^{2}=0$;

5. Sample Sizes: $N=1001 \mathrm{MZ}$ and DZ pairs, so with $T=5$ occasions, $D=20,020$ points.

This population structure is intended to mimic some aspects of a realistic longitudinal data collection, but it has far less complexity than real life data collections (i.e., linear slopes, no covariation, no incomplete data, and so on).

The biometric LCMs were fit to these data using SAS MIXED (Scripts 3 and 4) and NLMIXED programs in VCM form. The same models were fit using the latest versions of the Mplus and Mx programs in both PAM and VCM form. More complete computer scripts and the numerical results are presented in the Online Appendix, and a summary of results follows:

SEMA-PAM. The Mplus (and Mx) programs worked well and closely recovered all model parameters. The generating values were zero for some parameters, but sample values were often nonzero, and in some cases boundary restrictions on a variance term created a numerical problem. Even with these restrictions, the programs correctly characterize the population mechanism, and clearly point out the zero effects and the equally balanced effects. One obvious limitation of this SEMA programming is the lengthy code required $(\sim 100$ command lines). In Mplus, the use of the RANDOM command with TSCORE options made the coding simple, but extra code was devoted to overcoming the Mplus defaults (e.g., latent variables are correlated).

SEMA-VCM. This approach required very few changes from the Mplus (and Mx) programs, and has similar practical consequences. It was not necessary to use the reduced form equations to program this model because higher-order models can be programmed directly (i.e., Figure 4b). A simple combination of the basic equation and the common factor made this an easy alteration to the previous code. However, because the SEMA-VCM approach does not automatically create nonnegative (i.e., square root) variance restrictions, some negative variances were estimated for population parameters set at zero. In this case the
MLE are slightly closer to the population values than the SEMA-PAM versions. Other than the extensive and repetitive code required, this is also a reasonable way to fit these models.

MEMA-MIXED. Assuming the correct weighting scheme has been used (as in any univariate model, see Script 1), this was easily the most compact code to create and manipulate (Script 2). Furthermore, the MLE results are exactly identical to the SEM-VCM results, and the standard errors are extremely close. The fit $(-2 \mathrm{LL})$ is identical as well, and statistical decisions about the resulting 'significance of effects' does not differ from either SEM approach. There are several alternative ways of programming this model in SAS MIXED, and some of these require less computer memory (DDFM = SATTERTH; see Script 3b). The use of the Output Delivery System (SAS-ODS) allowed reasonably easy access to the estimated parameters, and facilitated subsequent calculation and plotting of parameter functions (Script 4).

MEMA-NLMIXED. These commands offered the most transparent and didactically appealing program script, and the easiest to extend to other more complex problems (e.g., McArdle et al., 2005). Unfortunately, this program did not appear to be able to converge in a reasonable amount of time. The preliminary results (after many hours) are not listed here, but the use of fast approximations (e.g., QPOINTS $=1$ or FIRO) may be necessary. Although this program worked well for the univariate cases (in McArdle \& Prescott, 2005), the practical utility of this way to program longitudinal models of reasonable complexity is not completely known.

\section{Application to Real Longitudinal Twin Data Selected SATSA Data}

The data used here includes nondemented twins from the SATSA (see Pedersen et al., 2002), a subset of twins from the population-based Swedish Twin Registry (Lichtenstein et al., 2002). The SATSA sample comprises all pairs of twins who indicated that they had been reared apart and separated before the age of 11 and a sample of twins reared together matched to the rearedapart group on gender, date and country of birth.

The SATSA data collection includes information on individuals at any time they were willing to participate. This leads to incomplete data over time for both complete and incomplete twin pairs. Individuals who met current diagnostic criteria for a diagnosis of dementia at any time during their participation in SATSA were excluded from this analysis (see Gatz et al., 1997). The data used here includes up to five waves of in-person testing covering a possible span of 16 years. Other studies (e.g., Finkel et al., 2003) have found substantial nonlinearity in some cognitive measures, especially before and after the age of 65 . To simplify these analyses, only data on individuals who were at least 65 years of age during their participation in SATSA are used. 
Table 1

Longitudinal Summary Statistics for the Information Subscale From the SATSA Data

\begin{tabular}{|c|c|c|c|c|c|}
\hline \multicolumn{6}{|c|}{ Means and deviations } \\
\hline Variable & $N$ & Mean & Std Dev & Minimum & Maximum \\
\hline Age[1] & 345 & 71.8 & 4.9 & 65.0 & 88.0 \\
\hline Age[2] & 339 & 72.3 & 5.3 & 65.0 & 91.0 \\
\hline Age[3] & 396 & 73.8 & 5.8 & 65.0 & 94.0 \\
\hline Age[4] & 354 & 76.3 & 6.8 & 65.0 & 96.4 \\
\hline Age[5] & 327 & 76.3 & 7.0 & 65.0 & 95.2 \\
\hline Info[1] & 344 & 67.2 & 20.0 & 13.6 & 100.0 \\
\hline $\operatorname{lnfo}[2]$ & 329 & 70.0 & 19.0 & 2.3 & 100.0 \\
\hline $\operatorname{lnfo[3]}$ & 385 & 70.2 & 19.0 & 0 & 100.0 \\
\hline $\operatorname{lnfo}[4]$ & 353 & 69.7 & 22.3 & 0 & 100.0 \\
\hline Info[5] & 327 & 74.2 & 20.2 & 0 & 100.0 \\
\hline \multicolumn{6}{|c|}{ Scale intercorrelations (and pairwise sample sizes) } \\
\hline & $\operatorname{lnfo}[1]$ & $\operatorname{lnfo}[2]$ & Info[3] & $\operatorname{lnfo}[4]$ & Info[5] \\
\hline \multirow[t]{2}{*}{ Info[1] } & 1.000 & & & & \\
\hline & (344) & & & & \\
\hline \multirow[t]{2}{*}{$\operatorname{lnfo}[2]$} & .890 & 1.000 & & & \\
\hline & $(234)$ & (329) & & & \\
\hline \multirow[t]{2}{*}{ Info[3] } & .870 & .881 & 1.000 & & \\
\hline & $(225)$ & $(281)$ & (385) & & \\
\hline \multirow[t]{2}{*}{$\operatorname{lnfo}[4]$} & .689 & .777 & .807 & 1.000 & \\
\hline & $(129)$ & (171) & (239) & (353) & \\
\hline \multirow[t]{2}{*}{$\operatorname{lnfo}[5]$} & .591 & .742 & .773 & .819 & 1.000 \\
\hline & (85) & $(116)$ & (172) & $(266)$ & (327) \\
\hline
\end{tabular}

Note: Age $[\mathrm{t}] \geq 65 ; T=4$ and $T=5$ were measured at the 5 th and 6 th visits.

Many cognitive domains are represented in the SATSA cognitive test battery (see Finkel et al., 2003), but only scores on the tests of Information are used here. This score indicated a factor of General Knowledge or Crystallized Intelligence and has not been found to decline rapidly over the adult life span (Horn, 1991). For ease of presentation this cognitive measure was rescaled to per cent correct of the total possible points (McArdle, 1988). Summary statistics for the SATSA data on chronological age and these
Information scores are presented in Table 1, and this is the same information presented earlier in Figure 1.

\section{Cross-Sectional Biometric Results}

The first series of analyses were based only on the cross-sectional data at Wave 1 on Information for $N=186$ twin pairs $($ Age[1] $\geq 65)$. The individual level data are not complete across twin pairs, so this analysis includes $N_{\mathrm{MZ}}=68$ persons with $D_{\mathrm{MZ}}=125$ scores, and $N_{\text {DZ }}=118$ persons with $D_{\mathrm{DZ}}=216$ scores. Biometric

Table 2

Initial Biometric Models for the Information Subscale From the SATSA Data

\begin{tabular}{|c|c|c|c|c|c|c|}
\hline \multirow{2}{*}{$\begin{array}{l}\text { Biometric } \\
\text { parameters } \\
\text { Fixed effects }\end{array}$} & \multicolumn{2}{|c|}{$\begin{array}{l}\text { (a) Full biometric decomposition } \\
(-2 \| l=2962, d f=341-6)\end{array}$} & \multicolumn{2}{|c|}{$\begin{array}{l}\text { (b) Revised biometric decomposition } \\
(-2 \|=2964, d f=341-5)\end{array}$} & \multicolumn{2}{|c|}{$\begin{array}{l}\text { (b) Revised biometric decomposition } \\
(-2 \| 1=2964, d f=341-4)\end{array}$} \\
\hline & MLE & (z value) & MLE & (zvalue) & MLE & (z value) \\
\hline$Y$ intercept @65 $\beta_{0}$ & 73.3 & (35.) & 72.8 & (34.) & 72.9 & (34.) \\
\hline$Y$ slope/decade $\beta_{1}$ & -8.3 & $(-3.3)$ & -8.0 & $(-3.1)$ & -8.0 & $(-3.1)$ \\
\hline \multicolumn{7}{|l|}{ Random effects } \\
\hline$A$-additive $\sigma_{0 a}^{2}$ & 211.0 & (2.3) & 209.1 & (2.3) & 225.3 & (5.7) \\
\hline$S$-shared $\sigma_{0 s}^{2}$ & 55.7 & $(0.7)$ & 14.5 & $(0.2)$ & $=0$ & $(-)$ \\
\hline$R$-rearing $\sigma_{0 r}^{2}$ & -76.2 & $(-1.2)$ & $=0$ & $(-)$ & $=0$ & $(-)$ \\
\hline E-environ. $\sigma_{0 e}^{2}$ & 159.3 & (5.7) & 160.7 & (5.3) & 158.3 & (5.8) \\
\hline
\end{tabular}

Note: Age $[t] \geq 65$ at Wave $T=1$ only, $N_{p}=186$ pairs. $D=341$ data points, Script $1+2$. 
parameters were estimated from the basic mixed-effects model (2) applied to all twin pairs at Wave 1 following the univariate coding presented in McArdle \& Prescott (2005, Script 1). One alteration of the biometric model made here was to take advantage of the SATSA design and add a parameter for the variance due to rearing environment $\left(\sigma_{r}^{2}\right)$. This new parameter was easily included in our input (see Script 1) by coding a new score (WeightR) with binary values reflecting individual rearing (i.e., $1=$ reared together and $0=$ reared apart). Since there are initial age differences between families, we also include age at the time of the first testing to account for this shared variation.

The numerical results are presented in Table 2 and Figure $5 \mathrm{a}$. In the first model (2a) all parameters were fitted and these results show some negative variance estimates (negative estimates were allowed using the NOBOUND option). In a second model (2b) the rearing environment parameter was deleted without much loss of fit $\left(\chi^{2}=2\right.$ on $\left.d f=1\right)$. In a third model (2c) the shared family component was deleted without much loss of fit $\left(\chi^{2}=0\right.$ on $\left.d f=1\right)$. The fixed parameters listed here include an intercept at Age $65\left(\beta_{0}=72.9\right)$, and a linear decline with age $\left(\sigma_{1}=-8.0\right.$ per decade). The variance parameters include the contribution of additive genetic effects $\left(\sigma_{a}^{2}=225.3,59 \%\right)$, no nonage related shared family genetic effects $\left(\sigma_{\mathrm{s}}^{2}=0\right)$, and substantial independent nonshared family genetic effects $\left(\sigma_{\mathrm{e}}^{2}=158.3,41 \%\right)$. These estimates do not include the common age related variance $(\sim 2 \%)$, and the nonshared variance is probably overestimated because it also includes all measurement error (McArdle \& Goldsmith, 1990).

\section{Longitudinal Growth Model Results}

Both the standard and orthogonal form of the LCM (see Figures 3) were fitted using SAS PROC MIXED software. This analysis included all only one twin from each family $(N=331)$ but raw data $(D=865)$ on as many time points available. The results presented in Table 3 are based on a mixed-effects algorithm with MLE, so they are considered optimal under standard MAR assumptions about the incomplete cases (e.g., Cnaan et al., 1997; McArdle \& Hamagami, 1992; Verbeke \& Molenberghs, 2000).

The first model ( $3 \mathrm{a})$ includes parameters representing independent level and slope parameters (see Script $3 \mathrm{a}$ ) and all parameters are accurate (at the $\alpha=.05$ test level). The fixed parameters include the expected mean at age $65\left(\beta_{0}=75.8\right)$ and the linear decline per decade $\left(\beta_{1}=-6.1\right)$. The first three variance components $\left(\sigma_{\mathrm{u} 0}{ }^{2}=332.0, \sigma_{\mathrm{u} 1}{ }^{2}=171.5, \sigma_{c 01}{ }^{2}=-44.5\right)$ are allowed to be negative (using NOBOUND) and these values are all reasonable. Following Equations 7, the results of the unrestricted growth model (Script 2a) can be calculated from the orthogonal model $\left(\sigma_{0}^{2}=\right.$ [332.0-44.5], $\left.\sigma_{1}^{2}=[171.5-44.5], \sigma_{01}=-44.5\right)$. The unique error variance $\left(\sigma_{u}^{2}=49.0\right)$ indicates sources of within time variance which are orthogonal over

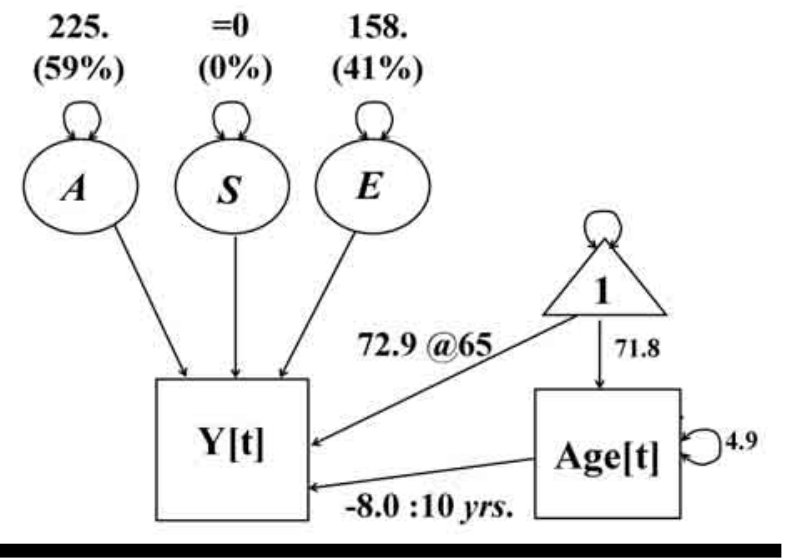

Figure 5a

Summary of biometric modeling results for Information from all twin data at Wave $1(N f=186 ; D=341)$.

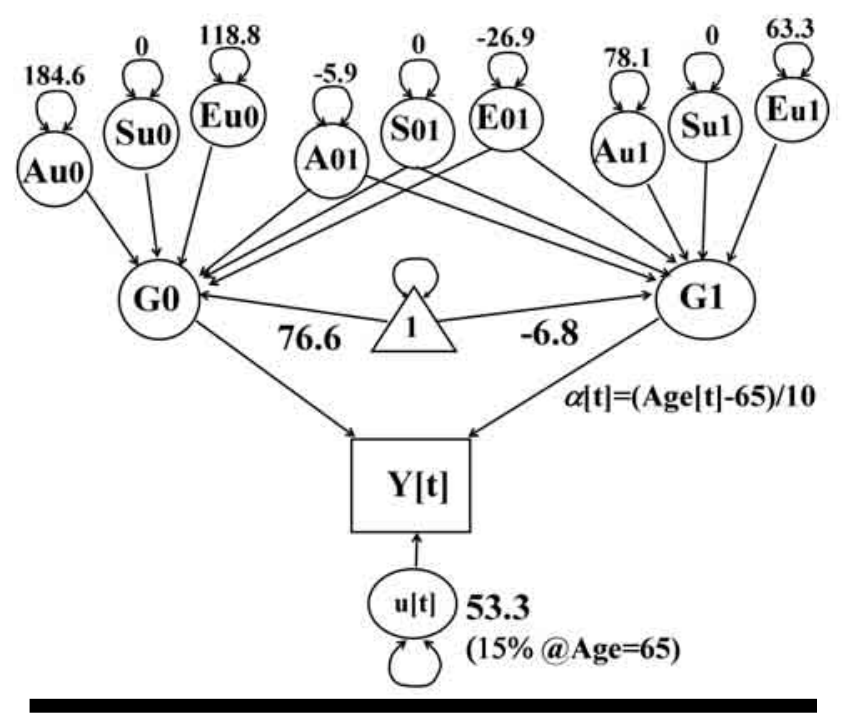

\section{Figure 5b}

Summary results for final biometric-longitudinal results from the twin latent curve model for Information over five occasions ( $N=360$ pairs; Age $\geq 65 ; D=1717$ ).

time. This estimation of the nonsystematic unique variance is the key feature which moves this otherwise simple linear model into the class of latent variable models and, and this estimate can be used to calculate the theoretical change in reliability of the growth measurement (see Equations 7; McArdle, 1986).

In the second model ( $3 \mathrm{~b})$ the two variance parameters associated with the slope $\left(\sigma_{\mathrm{u} 1}^{2}, \sigma_{\mathrm{c} 01}{ }^{2}\right)$ have been set to zero to yield a restricted model with individual differences in levels but not changes over time. These restrictions lead to a substantial loss of fit $\left(\chi^{2}=127\right.$ on $d f=2)$. In the third model $(4 \mathrm{c})$ the mean associated with the slope has been set to zero $\left(\beta_{1}=0\right)$ and this leads to another substantial loss of fit $\left(\chi^{2}=74\right.$ on $\left.d f=1\right)$. Using this final model as a baseline, these results suggest 
Table 3

Initial Longitudinal Models for the Information Scale From the SATSA Data

\begin{tabular}{|c|c|c|c|c|c|c|}
\hline \multirow{2}{*}{$\begin{array}{l}\text { Parameter } \\
\text { Fixed effects }\end{array}$} & \multicolumn{2}{|c|}{$\begin{array}{l}\text { a) Intercept and linear slope model } \\
(-2 \mid I=6916, d f=865-6)\end{array}$} & \multicolumn{2}{|c|}{$\begin{array}{l}\text { (b) Intercept and linear mean model } \\
(-2 \|=7043, d f=865-4)\end{array}$} & \multicolumn{2}{|c|}{$\begin{array}{l}\text { (b) Intercept only model } \\
(-2 \|=7117, d f=865-3)\end{array}$} \\
\hline & MLE & (z value) & MLE & (zvalue) & MLE & ( $z$ value $)$ \\
\hline G0-intercept @65 $\beta_{0}$ & 75.8 & (68.) & 75.0 & (65.) & 70.0 & (68.) \\
\hline G1-slope/decade $\beta_{1}$ & -6.1 & $(-6)$. & -6.2 & $(-9)$. & $=$ & $(-)$ \\
\hline \multicolumn{7}{|l|}{ Random effects } \\
\hline$U 0$-intercept $\sigma_{0}^{2}$ & 332.0 & (7.) & 291. & (11.) & 308.5 & (11.) \\
\hline U1-slope $\sigma_{1}^{2}$ & 171.5 & (4.9) & $=0$ & $(-)$ & $=0$ & $(-)$ \\
\hline U01-covar. $\sigma_{01}{ }^{2}$ & -44.5 & $(-2.2)$ & $=0$ & $(-)$ & $=0$ & $(-)$ \\
\hline$U[\mathrm{t}]$-unique $\sigma_{u}{ }^{2}$ & 49.0 & (13.) & 87.9 & (16.) & 97.0 & (16.) \\
\hline
\end{tabular}

Note: Age $[t] \geq 65 ; N=331$ individuals for up to $T=5$ and $D=865$; Script 3 .

there is substantial group and individual linear declines over age in Information scores.

\section{Longitudinal Biometric Latent Curve Results}

The longitudinal LCM was fitted to all twin data using mixed-effect techniques using SAS PROC MIXED software (see Figure 4b, Script 3). This analysis included all participants with any data at any time on either member of the pair. This selection resulted in 1717 data points of valid Information scores coming from $N_{\mathrm{MZ}}=123$ pairs with $D_{\mathrm{MZ}}=620$ scores, and $N_{\mathrm{DZ}}=208$ pairs with $D_{\mathrm{DZ}}=1097$ scores. The algorithm again used the MLE criterion, so these results are only appropriate under MAR assumptions about the incomplete cases for occasions and pairs. The numerical results are listed for three models in Table 4 and summarized in Figure $5 \mathrm{~b}$.

The first model (4a) is a full model with all MLE values estimated. While most of the parameters are meaningful, the lack of boundary conditions allowed

\section{Table 4}

Results of Longitudinal Biometric Modeling Using the Information Subscale Scores From SATSA Twins

\begin{tabular}{|c|c|c|c|c|c|c|}
\hline \multirow{2}{*}{$\begin{array}{l}\text { Parameter } \\
\text { Fixed effects }\end{array}$} & \multicolumn{2}{|c|}{$\begin{array}{l}\text { (a) Biometric intercept } \\
\text { and linear slope model } \\
(-2 \|=13642, d f=1717-15)\end{array}$} & \multicolumn{2}{|c|}{$\begin{array}{l}\text { (b) Revised biometric intercept } \\
\text { and linear slope model) } \\
(-2 \mid l=13647, d f=1717-12)\end{array}$} & \multicolumn{2}{|c|}{$\begin{array}{c}\text { (c) Revised biometric intercept } \\
\text { and linear slope } \\
(-2 \mid I=13647, d f=1717-9)\end{array}$} \\
\hline & MLE & ( $z$ value) & MLE & (zvalue) & MLE & ( $z$ value) \\
\hline G0-intercept @65 $\beta_{0}$ & 76.5 & (87.) & 76. & (89.) & 76.6 & (87.) \\
\hline $\begin{array}{l}\text { G1-slope/decade } \beta_{1} \\
\text { Random effects }\end{array}$ & \multicolumn{5}{|c|}{ Random effects } & $(-9)$. \\
\hline \multicolumn{7}{|l|}{$\begin{array}{l}\text { Additive genetic } \\
\text { components }\end{array}$} \\
\hline AU0-additive $\sigma_{0 a}^{2}$ & 343.4 & (2.3) & 166.2 & (2.0) & 184.6 & (3.2) \\
\hline AU1-additive $\sigma_{1 a}^{2}$ & 227.5 & (1.9) & 65.4 & $(1.0)$ & 78.1 & $(1.6)$ \\
\hline A01-additive $\sigma_{01 a}{ }^{2}$ & -100.2 & $(-1.5)$ & 2.0 & $(0.1)$ & -5.9 & $(-0.2)$ \\
\hline \multicolumn{7}{|l|}{ Shared environmental } \\
\hline SU0-shared $\sigma_{0 s}^{2}$ & -139.3 & $(-1.3)$ & $=0$ & $(-)$ & $=0$ & $(-)$ \\
\hline SU1-shared $\sigma_{1 s}{ }^{2}$ & -133.3 & $(-1.4)$ & $=0$ & $(-)$ & $=0$ & $(-)$ \\
\hline S01-shared $\sigma_{01 s}{ }^{2}$ & 89.1 & (1.8) & $=0$ & $(-)$ & $=0$ & $(-)$ \\
\hline \multicolumn{7}{|l|}{ Rearing environmental } \\
\hline RU0-rearing $\sigma_{0 r}{ }^{2}$ & 29.0 & (0.5) & 19.0 & $(0.3)$ & $=0$ & $(-)$ \\
\hline RU1-rearing $\sigma_{1 r}^{2}$ & 22.1 & $(0.5)$ & 11.7 & $(0.2)$ & $=0$ & $(-)$ \\
\hline R01-rearing $\sigma_{01 r}^{2}$ & 32.4 & $(0.8)$ & -7.8 & $(-0.3)$ & $=0$ & $(-)$ \\
\hline \multicolumn{7}{|l|}{ Unique environmental } \\
\hline EU0-environ. $\sigma_{0 e}{ }^{2}$ & 81.2 & (1.5) & 126.3 & (2.1) & 118.8 & (2.2) \\
\hline EU1-environ. $\sigma_{1 e}{ }^{2}$ & 32.4 & $(0.8)$ & 68.7 & (1.4) & 63.3 & (1.5) \\
\hline E01-environ. $\sigma_{01 e}{ }^{2}$ & -8.0 & $(-0.3)$ & -30.1 & $(-1.1)$ & -26.9 & $(-1.3)$ \\
\hline
\end{tabular}

Note: $N=360$ pairs for up to $T=5$ and $D=1717$; Script $1+4$. 


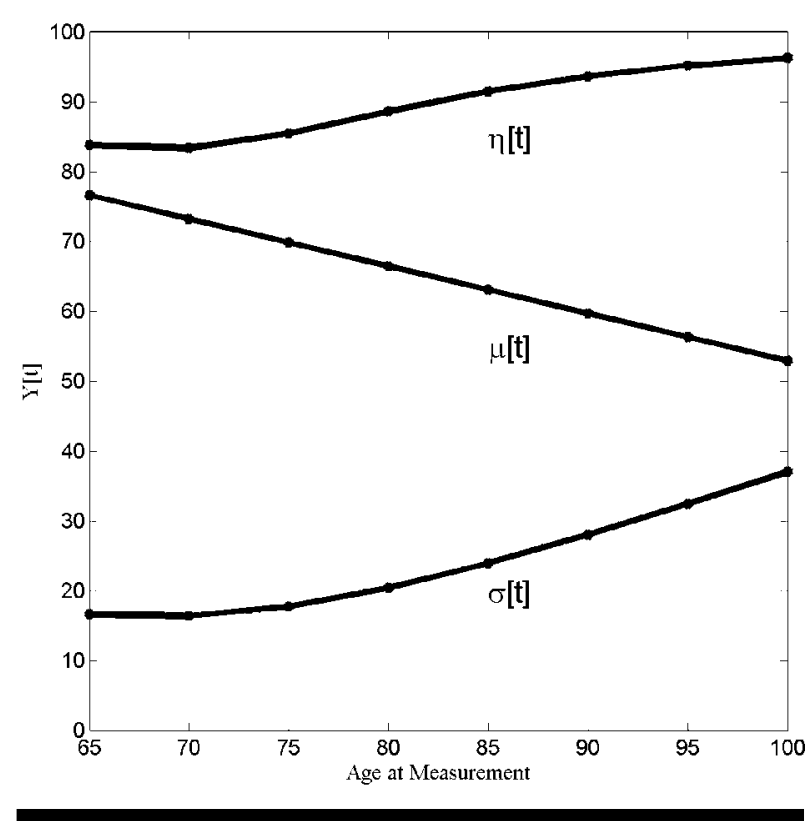

Figure 6a

Model based phenotypic curve expectations.

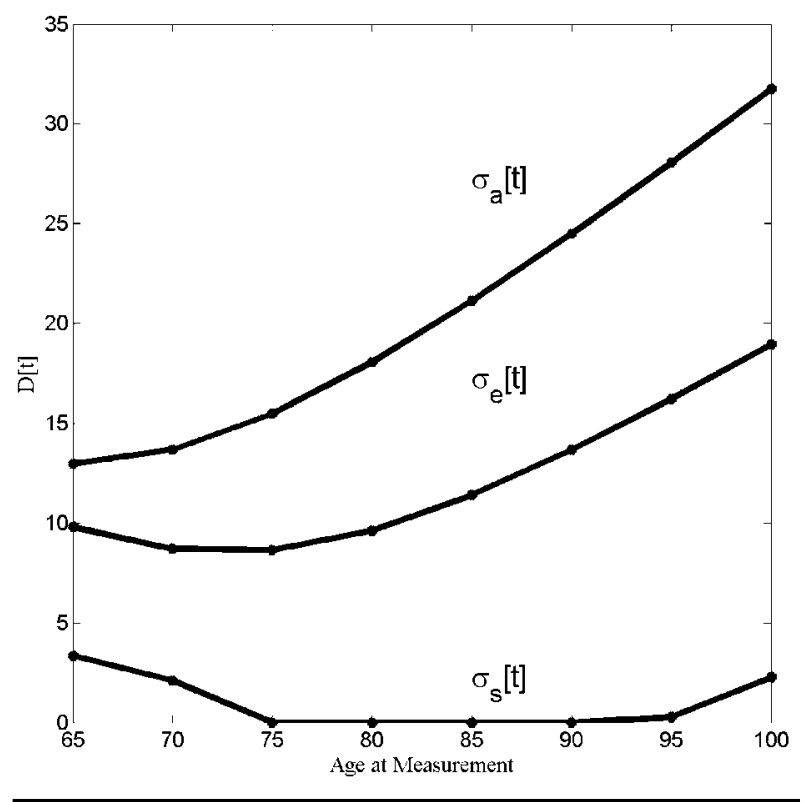

Figure 6b

Model based biometric curve model expectations.

several variance components to be large and negative. Even after the combination of the unique and common parts (as in Equations 7) some expected variances are negative, especially the shared environment parameters. In the second model $(4 \mathrm{~b})$ the shared environment parameters have been removed with only minor loss of fit $\left(\chi^{2}=5\right.$ on $\left.d f=3\right)$. In the third model $(4 c)$ the small parameters associated with the rearing environment were set to zero and again there is no loss of fit $\left(\chi^{2}=0\right.$ on $\left.d f=3\right)$. The parameters for the additive genetic and unique environment components are relatively large and cannot be removed without major loss of fit. Unlike the previous cross-sectional model (Table 2), here the unique environment terms contribute variance to the latent level and slope scores even after we account for the nonsystematic unique or error component $\left(\sigma_{u}^{2}=53.3\right)$.

It is difficult to obtain a complete understanding of the results of any biometric growth model only from these parameter estimates, and it is not appropriate to make inferences about the structure of the changes from tests based on any single parameter. To reinforce an old point, the parameter representing the "variance of the slope' is not equivalent to the resulting 'slope of the variance' (see McArdle, 1986; McArdle \& Hamagami, 2003; McArdle et al., 1998). One way we have dealt with this basic problem in prior research is to compare the changes in the expected variances at specific ages. This representation requires calculations such as Equation 13, and this was done using the simple SAS script (see Script 4). A table of the values resulting from these kinds of calculations is plotted in Figure 6.

The expected means $(\mu[\mathrm{t}])$ and deviations $(\sigma[\mathrm{t}])$ of the pheonotypic model parameters are plotted in Figure 6a and exhibit a declining mean with an increasing deviation. By adding the constant unique error term $\left(\sigma_{u}^{2}\right)$ we can also calculate and plot as the portion of the variance of the common scores at any time $(\eta[t])$. The phenotypic reliability is seen to rise over age.

The expectations for the biometric contributions can be used to calculate deviations for additive genetic $\left(\sigma_{a}[t]\right)$, shared environments $\left(\sigma_{s}[t]\right)$, and unique environments $\left(\sigma_{e}[t]\right)$. Since the shared environment parameter was fixed to zero for both the level and the slope, this remains at zero. In contrast, both the additive and environmental deviations are nonzero and increase over age (due to the slope and covariance). The additive component is a bit larger and gains a bit more. In turn, this implies the calculation of the percentage of variances within age has an increasing contribution of additive sources $\left(\eta_{\mathrm{a}[\mathrm{t}]}^{2}=.66\right.$ to .75$)$ and a necessarily decreasing contribution of environmental sources $\left(\eta_{\mathrm{e}[\mathrm{t}]}^{2}=.34\right.$ to .25$)$. Since the phenotypic variance over time is not constant, the percentage of variances over age may be misleading.

\section{$\overline{\text { Discussion }}$}

The purpose of this article was to present a new way of considering LCMs for longitudinal twin data. These initial simulation results demonstrate the adequacy and accuracy of the programming for MEMA and SEMA for well-structured longitudinal LCM twin analyses (except for NLMIXED). This is not surprising as McArdle and Prescott (2005) examined a wider range of bioimetric population models and found equivalent performances (also, see Ferrer et al., 2004). In order to further investigate the practical problems some of the simulated data were deleted using two different selection mechanisms: random dropout and 
nonrandom dropout (after McArdle \& Hamagami, 1992). In cases with incomplete data, both SEM and MEMA computer programs again produced identical estimates and fit to each other. In accordance with statistical theory, the selective loss of individuals and pairs was found to have major impacts on the results obtained, and extreme biases were found when only the complete cases were used. In this sense, the current programs work very well but cannot be expected to solve all contemporary longitudinal problems.

Other comparative aspects of these programs show advantages to using the MEMA approach. For example, to gain some idea about the changes to expect in the longitudinal models, we might be routinely interested in carrying out a form of repeated cross-sectional analysis. The same biometric cross-sectional analysis used here (Table 2) can be repeated for many different waves or for groups of similar age. Groups can be formed around specific ages, or age ranges, or even based sampling weights. To accomplish this in the MEMA framework, we can easily invoke the SAS Macro language and place a DO-Loop around the SAS PROC MIXED code. This repeated cross-sectional analysis is conceptually simple, but it is time consuming and practically difficult to run using SEM software not designed for general data analysis problems.

The substantive example based on SATSA Information scores showed approximately linear decline over this age range (age $\geq 65$ ). While the Information means decrease over age, the observed variance and genetic and environmental variance increased over age producing increasing heritability estimates. In more extensive SATSA research, Reynolds et al. (2002) and Finkel et al. (1998, 2003) examined sources of individual differences in cognitive levels and rate of change over time for three cognitive tests representing fluid ability, memory and perceptual speed domains. The general findings indicated larger genetic influences for the initial level while rate of change over time was influenced primarily by environmental factors not shared by twins. Similar findings have been suggested for general cognitive ability in Danish twins 70 years and older (Christensen et al., 2003; McGue \& Christensen, 2002). In contrast, McArdle et al. (1998) and McArdle and Hamagami (2003) report evidence of moderate to strong heritabilitities for change in Vocabulary and Block Design from the archival New York Twin study (NYT). One key difference in these studies is that the model fit was a model of change across waves-of-testing rather than age-of-testing as illustrated here. It is important to consider that wave-of-testing is often used as it is easier for SEM programs than age-at-testing. However, the results of LCMs based on time, even with linear slopes and baseline age as a predictor, are not strictly equivalent to LCM of age (McArdle et al., 2002). The MEMA approach presented here allows both approaches, so the investigator can makes this choice based on substantive reasons rather than practical limitations.

The MEMA approach can be extended for use with categorical outcomes (see McArdle \& Prescott, 2005), and this can be important in the context of longitudinal studies (see Yashin \& Iachine, 1995). Although not emphasized here, sample loss due to attrition (death and dysfunction) are likely to have led to initial underestimation of the genetic variance. Current SATSA research focuses on subgroup differences and accounting for dementia and other survival functions. MEMA models combining latent growth and survival models have been proposed (e.g., Faucett \& Thomas, 1996; Lin et al., 2002) and now standard software can be used for such analyses (i.e., NLMIXED, winBugs; see Eaves \& Erkanli, 2003; Guo \& Carlin, 2004; McArdle et al., 2005). Using these shared parameter models, future research will be able to examine the leading indicators of these cognitive transitions, including scores on cognitive tests administered at earlier ages, or indicators based on the latent level and latent slope of the key outcomes.

These MEMA approach presented here was designed to demonstrate a functionally useful methodological integration of developmental, genetic, and statistical concerns. While we must recognize the limitations of the standard LCM presented here, this approach easily generalizes to any longitudinal biometric analyses focused on the direct modeling of changes over time. For example, many useful nonlinear functions are fairly easy to consider under this framework (e.g., Equations 8; Pinheiro \& Bates, 2000; Cudeck \& Klebe, 2002). But much more can be accomplished within longitudinal twin and family analyses, including studies of incompleteness, considerations of latent classes, and the clarification of key hypotheses about sequential coupling (McArdle \& Hamagami, 2003). If we hope to achieve an improved understanding of individual and family development, then the last 20 years of work on the longitudinal twin analyses now need to evolve into new dynamic data collections and more substantively driven dynamic models. The MEMA approach presented here is only a small step in this direction.

\section{$\overline{\text { Acknowledgments }}$}

This research was supported by a grant from the National Institute on Aging (AG07137). The use of the Swedish Adoption/Twin Study of Aging (SATSA) was made possible by Dr. Nancy Pedersen, Karolinska Institute, and the data collection was supported by NIA (AG04563, AG10175), The MacArthur Foundation Research Network on Successful Aging, and the Swedish Council for Social Research (97:0147:1B). This work was conducted in the summer of 2004 while the author was a visiting scholar at the Harris-Manchester College of Oxford University. I thank my colleagues Fumiaki Hamagami, Carol Prescott, as well as Chandra Reynolds and an 
unknown reviewer, for their useful comments on earlier drafts of this work.

\section{Author Note}

Computer program input scripts are used in Appendix $A$ and can be found on the authors' website: http://www.usc.edu and search for 'McArdle'.

\section{References}

Baker, L. A., DeFries, J. C., \& Fulker, D. W. (1983). Longitudinal stability of cognitive ability in the Colorado Adoption Project. Child Development, 54, 290-297.

Boomsma, D. I., Martin, N. G., \& Molenaar, P. C. (1989). Factor and simplex models for repeated measures: Application to two psychomotor measures of alcohol sensitivity in twins. Behavior Genetics, 19, 79-96.

Bryk, A. S., \& Raudenbush, S. W. (1992). Hierarchical linear models: Applications and data analysis methods. Newbury Park, CA: SAGE.

Cattell, R. B. (1982). The inheritance of personality and ability: Research methods and findings. New York: Academic Press.

Christensen, K., Frederiksen, H., Vaupel, J. W., \& McGue, M. (2003). Age trajectories of genetic variance in physical functioning: A longitudinal study of Danish twins aged 70 years and older. Behavior Genetics, 33, 125-136.

Cnaan, A., Laird, N. M., \& Slasor, P. (1997). Using the general linear mixed model to analyse unbalanced repeated measures and longitudinal data. Statistics in Medicine, 16, 2349-2380.

Cudeck, R., \& Klebe, K. J. (2002). Multiphase mixedeffects models for repeated measures data. Psychological Methods, 7, 41-62.

Dolan, C. V., Molenaar, P. C. M., \& Boomsma, D. I. (1991). Longitudinal genetic analyses of longitudinal means and covariance structure using the simplex model in LISREL. Behavior Genetics, 21, 49-61.

Eaves, L., \& Erkanli, A. (2003). Markov Monte Carlo approaches to analysis of genetic and environmental components of human development change and G x E interaction. Behavior Genetics, 33, 279-299.

Faucett, C. L., \& Thomas, D.C. (1996). Simultaneously modeling censored survival data and repeatedly measured covariates: A Gibbs sampling approach. Statistics in Medicine, 15, 1663-1685.

Ferrer, E., Hamagami, F., \& McArdle, J. J. (2004). Modeling latent growth curves with incomplete data using different types of structural equation modeling and multilevel software. Structural Equation Modeling, 11, 452-483.

Finkel, D., Pedersen, N. L., Plomin, R., \& McClearn, G. E. (1998). Longitudinal and cross-sectional twin data on cognitive abilities in adulthood: The Swedish
Adoption/Twin Study of Aging. Developmental Psychology, 34, 1400-1413.

Finkel, D., Pedersen, N. L., Reynolds, C. A., Berg, S., de Faire, U., \& Svartengren, M. (2003). Genetic and environmental influences on decline in biobehavioral markers of aging. Behavior Genetics, Special Issue on Aging, 33, 107-123.

Finkel, D., Reynolds, C. A., McArdle, J. J., Gatz, M., \& Pedersen, N. L. (2003). Latent growth curve analyses of accelerating decline in cognitive abilities in late adulthood. Developmental Psychology, 39, 535-550.

Gatz, M., Pedersen, N. L., Berg, S., Johansson, B., Johansson, K., Mortimer, J. A., Posner, S. F., Viitanen, M., Winblad, B., \& Ahlbom, A. (1997). Heritability for Alzheimer's disease: The study of dementia in Swedish twins. Journals of Gerontology: Series A: Biological Sciences of Medical Sciences, 52, M117-M125.

Goldstein, H. (1995). Multilevel statistical models (2nd ed.). New York: Oxford Press.

Guo, G., \& Wang, J. (2002). The mixed or multilevel model for behavior genetic analysis. Behavior Genetics, 32, 37-49.

Guo, X., \& Carlin, B. P. (2004). Separate and joint modeling of longitudinal and event time data using standard computer packages. American Statistician, $58,1-9$.

Horn, J. L. (1991). Measurement of intellectual capabilities: A review of theory. In K. S. McGrew, J. K. Werder, \& R. W. Woodcock, Woodcock-Johnson technical manual (pp. 197-246). Allen, TX: DLM Teaching Resources.

Johansson, B., Hofer, S. M., Allaire, J. C., MaldonadoMolina, M. M., Piccinin, A. M., Berg, S., Pedersen, N. L., \& McClearn, G. E. (2004). Change in cognitive capabilities in the oldest old: The effects of proximity to death in genetically related individuals over a 6year period. Psychology and Aging, 19, 145-156.

Jöreskog, K. G., \& Sörbom, D. (1984). LISREL VII: A guide to the program and applications. Chicago: SPSS Inc.

Lichtenstein, P., deFaire, U., Floderus, B., Svartengren, M., Svedberg, P., \& Pedersen, N. L. (2002). The Swedish Twin Registry: A unique resource for clinical, epidemiological and genetic studies. Journal of Internal Medicine, 252, 184-205.

Lin, H., McCulloch, C. E., \& Mayne, S. T. (2002). Maximum likelihood estimation on the joint analysis of time-to-event and multiple longitudinal variables. Statistics in Medicine, 21, 2639-2382.

Littell, R. C., Milliken, G. A., Stroup, W. W., \& Wolfinger, R. D. (1996). SAS system for mixed models. Cary, NC: SAS Institute.

McArdle, J. J. (1986). Latent variable growth within behavior genetic models. Behavior Genetics, 16, 163-200. 
McArdle, J. J. (1988). Dynamic but structural equation modeling of repeated measures data. In J. R. Nesselroade \& R. B. Cattell (Eds.), The handbook of multivariate experimental psychology (Vol. 2, pp. 561-614). New York: Plenum Press.

McArdle, J. J., \& Bell, R. Q. (2000). Recent trends in modeling longitudinal data by latent growth curve methods. In T. D. Little, K. U. Schnabel, \& J. Baumert (Eds.), Modeling longitudinal and multiple-group data: Practical issues, applied approaches, and scientific examples (pp. 69-108). Mahwah, NJ: Erlbaum.

McArdle, J. J., Ferrer-Caja, E., Hamagami, F., \& Woodcock, R.W. (2002). Comparative longitudinal multilevel structural analyses of the growth and decline of multiple intellectual abilities over the lifespan. Developmental Psychology, 38, 115-142.

McArdle, J. J., \& Goldsmith, H. H. (1990). Some alternative structural equation models for multivariate biometric analyses. Behavior Genetics, 20, 569-608.

McArdle, J. J., \& Hamagami, F. (1992). Modeling incomplete longitudinal and cross-sectional data using latent growth structural models. Experimental Aging Research, 18, 145-166.

McArdle, J. J., \& Hamagami, F. (1996). Multilevel models from a multiple group structural equation perspective. In G. Marcoulides \& R. Schumacker (Eds.), Advanced structural equation modeling: Issues and techniques (pp. 89-124). Hillsdale, NJ: Erlbaum.

McArdle, J. J., \& Hamagami, F. (2003). Structural equation models for evaluating dynamic concepts within longitudinal twin analyses. Behavior Genetics, 33, 137-159.

McArdle, J. J., \& Prescott, C. A. (2005). Mixed-effects variance components models for biometric family analyses. Behavior Genetics, 35, 631-652.

McArdle, J. J., Prescott, C. A., Hamagami, F., \& Horn, J. L. (1998). A contemporary method for developmentalgenetic analyses of age changes in intellectual abilities. Developmental Neuropsychology, 14, 69-114.

McArdle, J. J., Small, B. J., Backman, L., \& Fratiglioni, L. (2005). Longitudinal models of growth and survival applied to the early detection of Alzheimer's disease. Journal of Geriatric Psychiatry and Neurology, 18, 234-241.

McArdle, J. J., \& Woodcock, R. W. (1997). Expanding test-retest designs to include developmental time-lag components. Psychological Methods, 2, 403-435.

McGue, M., \& Christensen, K. (2002). The heritability of level and rate-of-change in cognitive functioning in
Danish twins aged 70 years and older. Experimental Aging Research, 28, 435-451.

Muthén, L. K., \& Muthén, B. O. (2002). Mplus, the comprehensive modeling program for applied researchers user's guide. Los Angeles, CA: Muthén \& Muthén.

Neale, M. C., Boker, S. M., Xie, G., \& Maes, H. H. (1999). Mx: Statistical modeling (5th ed.). VCU Box 900126, Richmond, VA: Department of Psychiatry.

Neale, M. C., \& McArdle, J. J. (2000). Structured latent growth curves for twin data. Twin Research, 3, 1-13.

Pedersen, N. L., Lichtenstein, P., \& Svedberg, P. (2002). The Swedish Twin Registry in the third millennium. Twin Research, 5, 427-432.

Pedersen, N. L., Ripatti, S., Berg, S., Reynolds, C., Hofer, S. M., Finkel, D.,Gatz, M., \& Palmgren, J. (2003). The influence of mortality on twin models of change: Addressing missingness through multiple imputation. Behavior Genetics, 33, 161-169.

Pinheiro, J. C., \& Bates, D. M. (2000). Mixed-effects models in $S$ and S-PLUS. New York: Springer.

Prescott, C. A. (2004). Using the Mplus computer program to estimate models for continuous and categorical data from twins. Behavior Genetics, 1, 17-40.

Reynolds, C. A., Finkel, D., Gatz, M., \& Pedersen, N. L. (2002). Sources of influence on rate of cognitive change over time in Swedish twins: An application of latent growth models. Experimental Aging Research, $28,407-433$.

Reynolds, C. A., Finkel, D., McArdle, J. J., Gatz, M., Berg, S., \& Pedersen, N. L. (2005). Quantitative genetic analysis of latent growth curve models of cognitive abilities in adulthood. Developmental Psychology, 41, 3-16.

Reynolds, C. A., Gatz, M., \& Pedersen, N. L. (2002). Individual variation for cognitive decline: Quantitative methods for describing patterns of change. Psychology and Aging, 17, 271-287.

SAS (1999). SAS (Version 8.1). Cary, NC: SAS Institute, Inc.

Vandenberg, S. G., \& Falkner, F. (1965). Heredity factors in human growth. Human Biology, 37, 357-365.

Verbeke, G., \& Molenberghs, G. (2000). Linear mixed models for longitudinal data. New York: Springer.

Yashin, A. I., \& Iachine, I. A. (1995). Genetic analysis of durations: Correlated frailty model applied to survival of Danish twins. Genetic Epidemiology, 12, 529-538. 


\section{$\overline{\text { Appendix A }}$}

\section{Input Scripts for Longitudinal Biometric Analysis With SAS MIXED see http://www.usc.edu and search for 'McArdle'}

\section{Script 1: SAS DATA script to Create Appropriate Weights for each Pair Type}

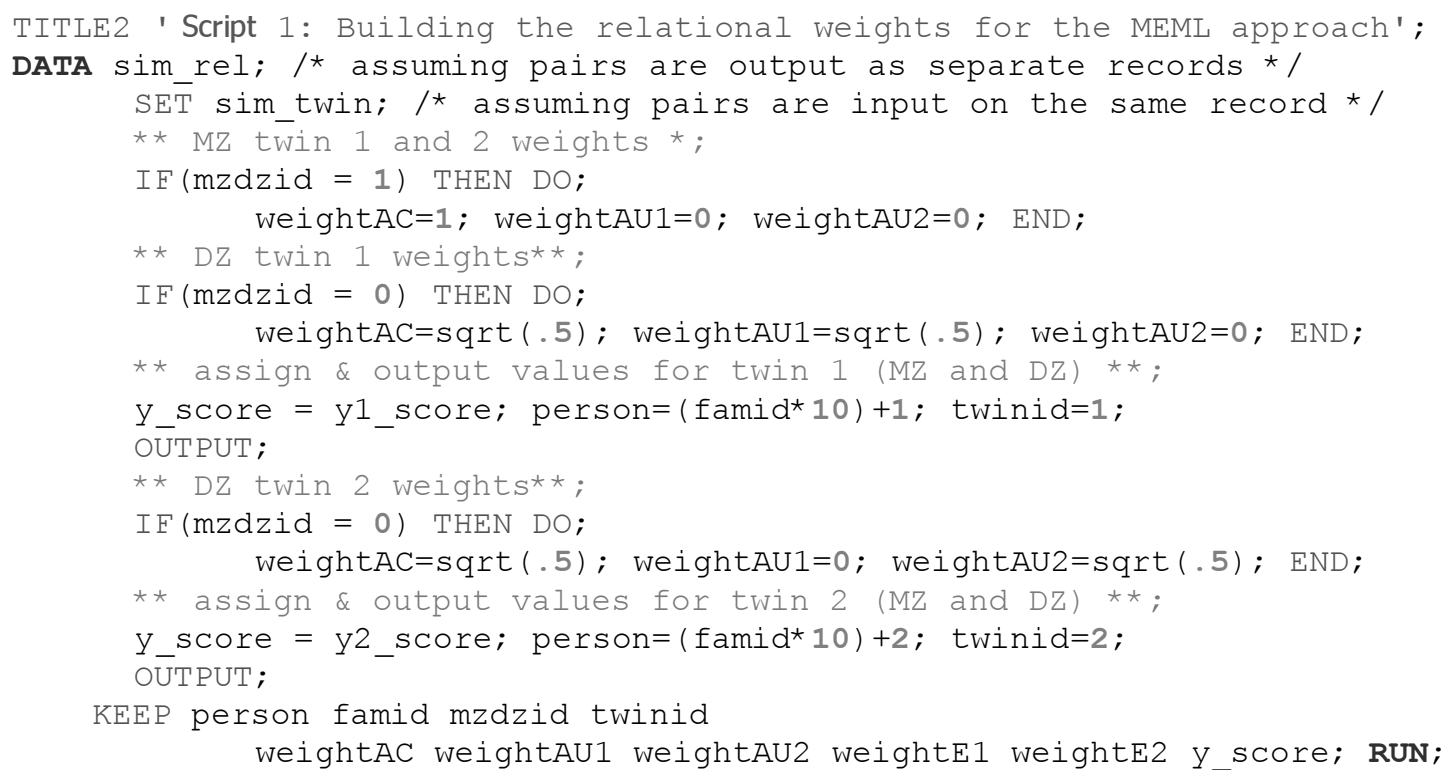

\section{Script 2: SAS PROC MIXED script to Calculate Biometric Model Estimates}

TITLE2 'Script 2: Biometric VC Approach 1 - Twinid and Famid approach with NOBOUNDS';

PROC MIXED NOCLPRINT COVTEST METHOD=ML; CLASS twinid famid ;

MODEL yt = / SOLUTION DDFM= DDFM=SATTERTH CHISQ ; $/ *$ Mean and E Variance for y0 $* /$ RANDOM INTERCEPT / SUBJECT=famid TYPE=VC; / $\mathrm{C}$ for $\mathrm{y} 0 *$ /

RANDOM weightAC weightAU1 weightAU2 / SUBJECT=famid TYPE=TOEP(1); /* A for y0 */ PARMS / NOBOUND; RUN;

\section{Script 3: SAS MIXED scripts for the Growth models as Variance Components}

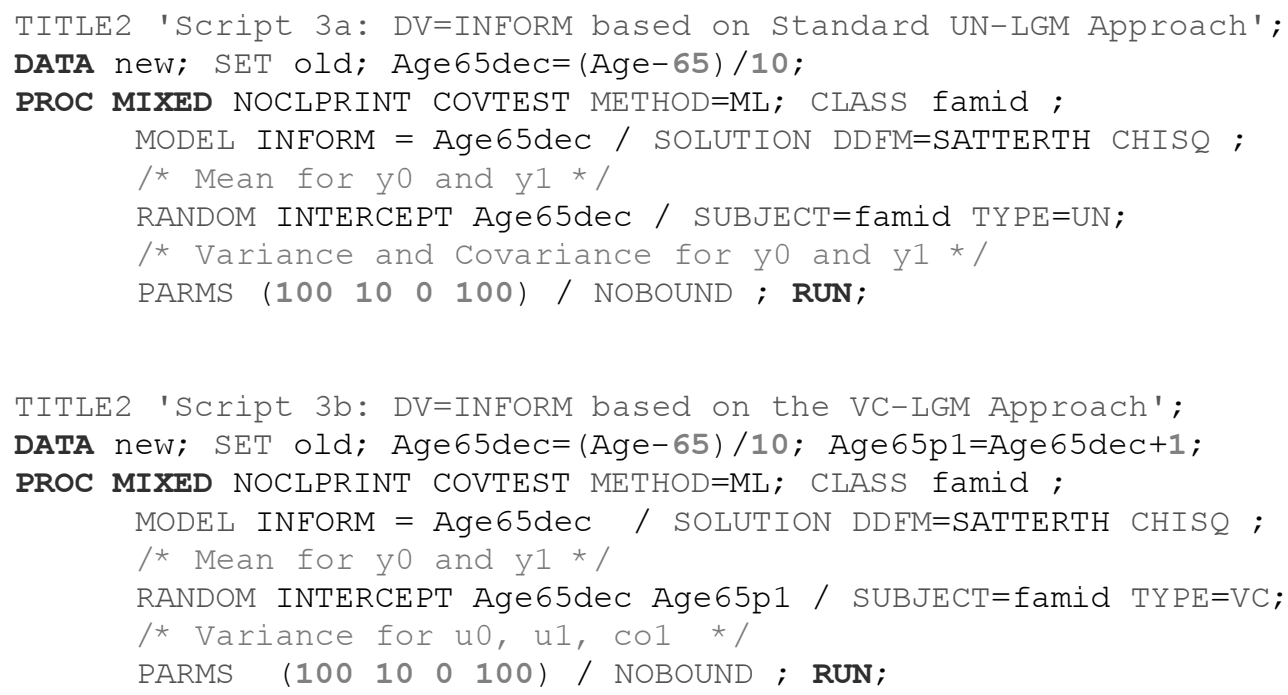




\section{Script 4: SAS MIXED scripts for the Longitudinal-Biometric models}

TITLE2 'Script 4a: VC Approach 1 - Twinid and Famid approach with NOBOUNDS';

PROC MIXED DATA=sim_dyn5 NOCLPRINT COVTEST METHOD=ML; CLASS twinid famid ; MODEL yt = age / SOLUTION DDFM=SATTERTH CHISQ ;

/* Mean for $\mathrm{y} 0$ and $\mathrm{y} 1 * /$

RANDOM INTERCEPT age agep1 / SUBJECT=famid(twinid) TYPE=VC;

/* E for u0, u1, c01*/

RANDOM INTERCEPT age agep1 / SUBJECT=famid TYPE=VC;

/* C for u0, u1, c01*/

RANDOM weightAC weightAU1 weightAU2 / SUBJECT=famid TYPE=TOEP(1);

/* A for y0 */

RANDOM weightAC*age weightAU1*age weightAU2*age / SUBJECT=famid TYPE=TOEP(1);

/* A for $\mathrm{y} 1 * /$

RANDOM weightAC*agep1 weightAU1*agep1 weightAU2*agep1 / SUBJECT=famid TYPE=TOEP(1);

/* A for $\mathrm{C} 01 *$ /

PARMS / NOBOUND;

RUN;

TITLE2 'Script 4b: VC Approach 3 - Twinid and Famid approach with NOBOUNDS and Restrictions';

PROC MIXED DATA=sim_dyn5 NOCLPRINT COVTEST METHOD=ML; CLASS twinid famid ;

MODEL yt = age / SOLUTION DDFM= DDFM=SATTERTH CHISQ ;

/* Mean for $y 0$ and $y 1 * /$

RANDOM INTERCEPT age agep1 / SUBJECT=famid(twinid) TYPE=VC;

/*E for $\mathrm{u} 0, \mathrm{u} 1, \mathrm{C} 01 * /$

RANDOM INTERCEPT age agep1 / SUBJECT=famid TYPE=VC;

/* C for u0, u1, c01*/

RANDOM weightAC weightAU1 weightAU2 / SUBJECT=famid TYPE=TOEP(1);

/* A for u0 */

RANDOM weightAC*age weightAU1*age weightAU2*age / SUBJECT=famid TYPE=TOEP(1);

/* A for u1 */

RANDOM weightAC*agep1 weightAU1*agep1 weightAU2*agep1 / SUBJECT=famid TYPE=TOEP(1);

/*A for $\mathrm{CO} * /$

PARMS (100 100000100100 100) / EQCONS=4,5, 6 NOBOUND ; RUN; 
